\title{
"¡Abre los ojos, un puente!" o cómo tomar conciencia de la importancia de la ingeniería mientras se aprende un idioma extranjero
}

Ignacio Payá-Zaforteza ${ }^{a}$, Mariana Bono ${ }^{b}$

${ }^{a}$ Princeton University, Dep. of Civil and Environmental Engineering - Universitat Politècnica de València, Dep. de Ingeniería de la Construcción y Proyectos de Ingeniería Civil, email: igpaza@cst.upv.es , ${ }^{\mathrm{b}}$ Princeton University, Dept. of Spanish and Portuguese Languages and Cultures, email: $\underline{\text { mbono@princeton.edu } . ~}$

\begin{abstract}
Structural engineering is commonly seen as a purely technical discipline. However, this should not be the case because engineering is much more than technique. Changing this common view requires the development of activities that open the eyes of the students (engineers and non-engineers) to a view of engineering as a very creative process.

Within this context, this paper presents a new experience developed at Princeton University in an advanced course on Spanish language and culture. This experience is the result of the collaboration of a professor in civil enginnering with a professor of Spanish language and culture and enabled the students to: (1) increase their awareness of engineering as a creative discipline, (2)learn the basics of structural engineering through the construction of simple models of bridges, (3) enlarge their vocabulary, (4) improve their oral comprenhension and (5) practice note taking in an environment similar to the one of a scientific conference or a professional meeting. From the professors' point of view, the experience has been very enriching and has set the foundation for future collaborations combining foreing language learning, engineering and art.
\end{abstract}

Keywords: multidisciplinary collaboration; engineering; society; art; Spanish; bridges 
"¡Abre los ojos, un puente!” o cómo tomar conciencia de la importancia de la ingeniería mientras se aprende un idioma extranjero

\begin{abstract}
Resumen
La ingeniería estructural es generalmente vista como algo principalmente técnico. Sin embargo, esto no debería ser así porque la ingeniería es más que técnica. Cambiar esta visión plantear actividades que abran los ojos de los futuros profesionales (ingenieros y no ingenieros) y les permitan descubrir la parte creativa de la ingeniería.

En este contexto, esta ponencia presenta una experiencia realizada en un curso oficial de nivel avanzado de español en la Princeton University de los USA. La experiencia es el fruto de una colaboración de un profesor del área de ingeniería estructural con una profesora del área de lengua y cultura española y ha conseguido que los alumnos: (1) tomen conciencia de los valores estéticos y sociales de la ingeniería estructural, (2) aprendan los fundamentos de la ingeniería estructural de forma activa realizando modelos reducidos de puentes, (3) desarrollen competencias como la ampliación del léxico disponible y la mejora de la comprensión oral en español y la toma de apuntes como se hace en una reunión o conferencia. Para los profesores, la experiencia ha supuesto un enriquecimiento mutuo y un punto de partida para experiencias docentes más complejas que integren el aprendizaje de un idioma extranjero con la ingeniería y el arte.
\end{abstract}

Palabras clave: colaboración multidisciplinar; ingeniería; sociedad; arte; español; puentes.

\title{
1. Introducción
}

La ingeniería estructural que posibilita la realización de puentes y edificios es vista normalmente como una disciplina estrictamente técnica. Por ello, es habitual que tanto el público general como los propios ingenieros desconozcan las posibilidades de expresión artística que tiene la ingeniería estructural y la creatividad que esconden los proyectos de ingeniería. Como consecuencia de ello:

(a) La búsqueda de la belleza puede convertirse en un objetivo casi absoluto, no equilibrado por la búsqueda de la economía y de la eficiencia en el uso de los recursos (véase la Fig. 1 y Sobrino, 2013). Esta visión puede ser peligrosa para los ciudadanos que, con sus impuestos, financian las obras públicas.

(b) La búsqueda de la economía puede acabar produciendo diseños muy funcionales y económicos, pero con una estética e impacto visual desastrosos (véase la Fig. 2).

(c)) EY-NC-ND 2015, Universitat Politècnica de València

Congreso IN-RED (2015) 
Sin embargo, es posible encontrar soluciones que combinan de forma equilibrada economía, eficiencia y elegancia. Un ejemplo de una de ellas puede verse en la Fig. 3 y un estudio detallado de la historia de la ingeniería estructural desde esta óptica puede encontrarse en Billington $(1985,2013)$. Para que la sociedad prefiera y exija soluciones equilibradas es muy importante la realización de actividades divulgativas que expliquen de forma sencilla algunos de los mejores ejemplos de ingeniería estructural.

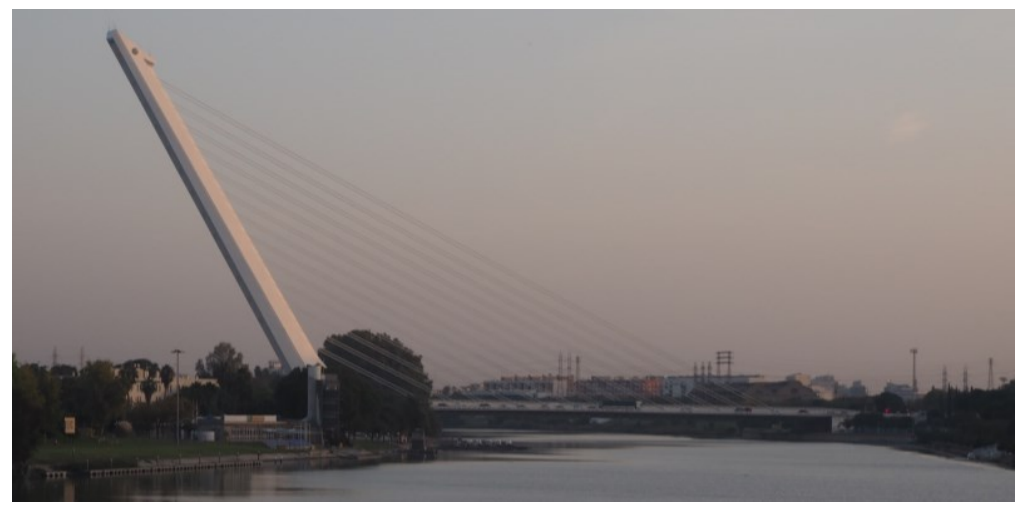

Fig. 1. Puente del Alamillo en Sevilla (1992). Proyectista: S. Calatrava. Imagen: I. Payá Zaforteza.

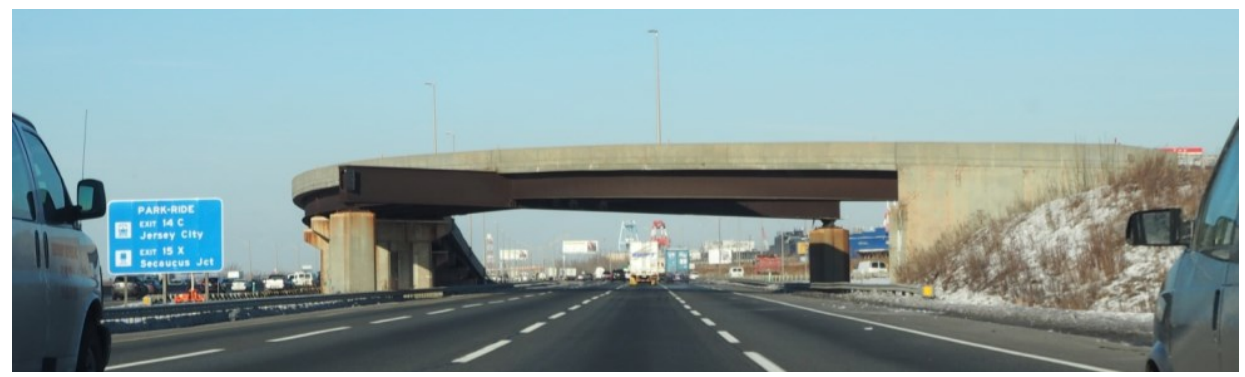

Fig. 2. Paso superior en Nueva Jersey, USA. Ejemplo de estructura poco atractiva visualmente. Imagen: I. Payá Zaforteza.

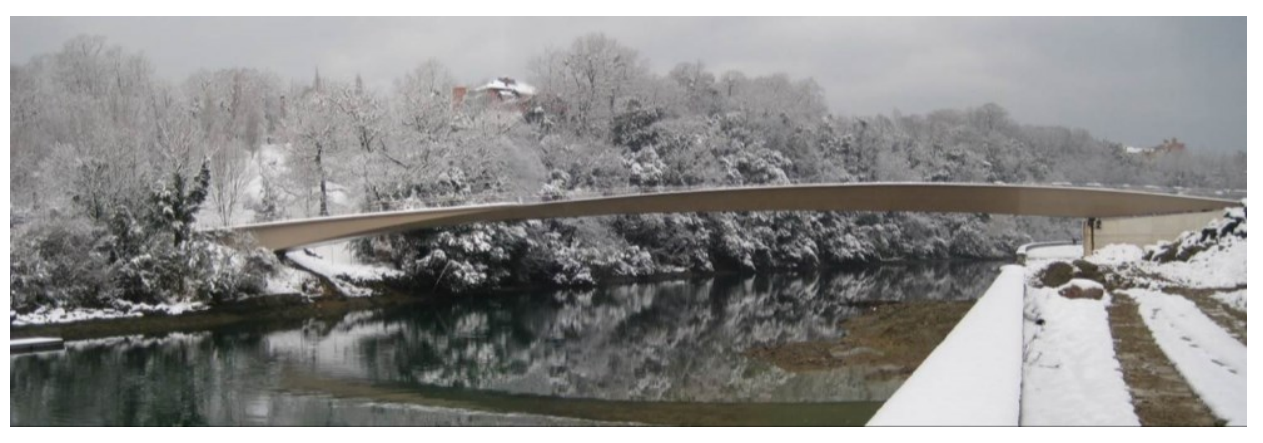

Fig. 3. Pasarela Mikel Laboa en San Sebastián, ejemplo de una estructura que combina economía, eficiencia y elegancia. Ing. Mario Guisasola. Imagen: M. Guisasola.

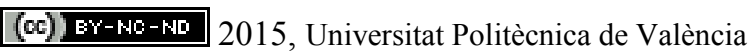

Congreso In-Red (2015) 
"'Abre los ojos, un puente!" o cómo tomar conciencia de la importancia de la ingeniería mientras se aprende un idioma extranjero

En este contexto general, esta ponencia explica la colaboración entre el Department of Civil and Environmental Engineering y el Department of Spanish and Portuguese Languages and Cultures de la Princeton University de los Estados Unidos. La colaboración divulga la importancia de la ingeniería estructural mientras contribuye al aprendizaje de un idioma extranjero. La ponencia empieza explicando el contexto y objetivos de la actividad, continua detallando su desarrollo y resultados obtenidos y termina explicando las principales conclusiones del trabajo realizado.

\section{Contexto de la actividad}

La actividad que se presenta se realizó con alumnos de la asignatura SPA-207. Studies in Spanish Language and Style. Este es un curso de lengua española de nivel avanzado que se oferta a los estudiantes de Princeton con un enfoque temático innovador alineado con las mejores prácticas pedagógicas preconizadas por la Modern Language Association (2007) y el American Council for the Teaching of Foreign Languages (National Standards in Foreign Language Education Project, 2006), entre otros. Aproximadamente 50 estudiantes se inscriben en este curso cada semestre y cada clase o sección está limitada a 12 estudiantes. Los alumnos proceden tanto del ámbito científico-técnico como de las humanidades.

El principal objetivo buscado al diseñar el curso fue dotarlo de un aspecto a menudo ausente en las clases de lenguas, a saber, contenidos culturales sólidos y consecuentes. El énfasis en la comunicación y en las habilidades funcionales que caracterizan a las metodologías dominantes en la didáctica de lenguas no debe hacer perder de vista que la comunicación nunca está libre de presupuestos culturales. Por otra parte, el lenguaje tiene un papel fundamental en la negociación local del sentido y en la construcción de narrativas que son representativas de una cultura. Estos aspectos críticos del lenguaje y la comunicación a menudo se pierden en los cursos de lengua que solo ofrecen una colección aleatoria de lecturas, audios y unidades gramaticales que terminan creando una visión normativa, esencialista y monolítica sobre la lengua y las culturas hispanas.

Para alcanzar este objetivo, se utiliza la ciudad como microcosmo tangible de prácticas lingüísticas y culturales histórica y geográficamente situadas. Las grandes ciudades son un concentrado de la riqueza material y simbólica de un país, motores de creación artística y cultural, sitios en los que arte y cultura se cargan de sentido. La ciudad, al igual que la lengua, refleja la cultura: un mundo de significaciones compartidas. Los contenidos de este curso giran entorno a dos grandes capitales del mundo hispanohablante, Madrid y Buenos Aires. Los estudiantes desarrollan sus competencias lingüísticas al mismo tiempo que exploran el paisaje urbano, la producción artística y literaria, la actualidad y la experiencia diaria de porteños y madrileños en las calles de su ciudad.

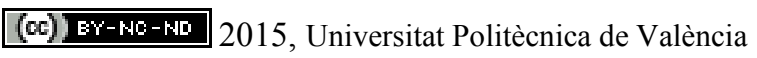

Congreso IN-RED (2015) 
La ciudad da un marco concreto pero flexible, vibrante, diverso, moderno, que celebra el cambio y la reinvención y que permite introducir la complejidad en el currículo. Este enfoque permite ampliar el concepto de lo que se enseña en la clase de lengua para abarcar los discursos públicos de la sociedad hispanohablante en el ámbito de la educación y la investigación, el trabajo y las profesiones, las artes y la sociedad civil (Byrnes, 2002).

Las principales prácticas pedagógicas empleadas en el curso son:

- Apertura hacia e inclusión de otras disciplinas: los estudiantes exploran las artes, las ciencias, las profesiones a través de visitas a museos, proyectos de colaboración interdepartamentales, charlas con conferenciantes invitados, etc.

- Enfasis en la creación y la producción original: inclusión de performances, teatro, escritura creativa, producción audiovidual, portfolios digitales, etc.;

- Prácticas autoreflexivas: reflexión metalingüística (hablar la lengua y hablar de la lengua, de sus variedades dialectales, de la relación entre lengua y poder), metacognitiva (¿cómo aprendemos?), socioafectiva (¿por qué? ¿para qué?);

- Servicio a la comunidad: creación de vínculos con asociaciones y ONG locales, emprendimientos culturales o educativos para insertar el aprendizaje en marcos que van más allá de la institución educativa.

Sería fácil crear un curso sobre Madrid y enseñarlo en Madrid o sobre Buenos Aires en Buenos Aires. En Princeton, el desafío es crear proximidad donde no la hay, traer la ciudad a la clase, recrear un cierto ambiente urbano, las idiosincrasias que distinguen estas ciudades de otras. Está claro que hay que ir más allá de la postal, de la mirada romántica del turista, de los estereotipos y lugares comunes. Una manera de lograrlo es creando proyectos que ocupan a los estudiantes durante varias semanas, que los hacen interactuar con gente y/o artefactos artísticos y culturales y que les dan agencia, control sobre los contenidos, poder de decisión y acceso directo a las narrativas que otra cultura crea sobre sí misma. Algunos ejemplos de estos proyectos son:

El museo como aula: los museos dentro y alrededor de los campus estadounidenses ofrecen la posibilidad de contacto directo con obras producidas fuera. Hace unos meses el Museo de Arte de Princeton presentó una exposición fantástica llamada The itinerant languages of photography, que incluyó trabajos de Gian Paolo Minelli, un fotógrafo suizo basado en Buenos Aires. Se organizó un taller de escritura en las galerías del museo, seguido por un trabajo de composición en el que los estudiantes debían describir y ofrecer una respuesta crítica al material fotográfico. Con este trabajo, se puede desarrollar la habilidad de los estudiantes para elaborar textos descriptivos (hay todo un trabajo previo sobre el adjetivo, las cláusulas relativas, la comparación en la descripción) al mismo tiempo que se les alienta a interpretar una cultura visual de manera sumamente original e informada.

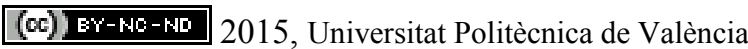


"¡Abre los ojos, un puente!" o cómo tomar conciencia de la importancia de la ingeniería mientras se aprende un idioma extranjero

Portfolios digitales Barrios de Madrid y Buenos Aires: los estudiantes utilizan la plataforma VoiceThread para crear y compartir portfolios que trazan un recorrido por algunos barrios de Madrid y Buenos Aires. Esta actividad se lleva a cabo en grupos y busca animar a los estudiantes a investigar y seleccionar una variedad de contenidos (texto, video, imagen) representativos de cada barrio, añadiendo comentarios para analizar la importancia o interés de las fuentes seleccionadas. El objetivo principal es crear una conversación dentro y fuera de la clase, con contenidos determinados por los propios estudiantes.

\section{Objetivos de la innovación}

Los principales objetivos de la colaboración desarrollada fueron:

- Reforzar la adquisición de competencias lingüísticas en un idioma extranjero (en este caso, el español) de forma activa y entretenida.

- Poner de manifiesto el valor de los puentes como expresiones culturales de nuestra sociedad.

- Conectar la enseñanza de la ingeniería con la enseñanza de las humanidades.

- Divulgar, conseguir que alumnos universitarios de diferentes ramas de conocimiento (y la mayoría no procedentes de enseñanzas técnicas) tomen conciencia de la importancia de la ingeniería estructural y aprendan algunos de sus conceptos básicos.

- Desarrollar la capacidad de crítica y contribuir a la formación de una ciudadanía mejor informada.

\section{Desarrollo de la innovación}

La actividad se desarrolló en una sesión de 50 minutos siguiendo las fases siguientes:

a) Fase 1. Los alumnos realizaron un cuestionario empleando sus ordenadores o teléfonos inteligentes mediante la aplicación "Kahoot". El cuestionario buscaba iniciar la reflexión de los alumnos hacia la consideración de la ingeniería de puentes como arte a partir de preguntas como "¿Pueden ser los puentes obras de arte?” ¿Quién es responsable de diseñar los puentes?” ¿Qué valoración darías a este puente?”, “Son los puentes bonitos caros?”

Los resultados de esta encuesta demostraron que los estudiantes:

- Estaban de acuerdo en que los puentes son importantes para la sociedad.

- Consideraban que los ingenieros eran responsables de diseñar los puentes. Sin embargo, cuando la pregunta se matizaba y se preguntaba quién era responsable de hacer los puentes bonitos, un $76 \%$ indicaba que eran los arquitectos y solo un $24 \%$ indicaba que los ingenieros.

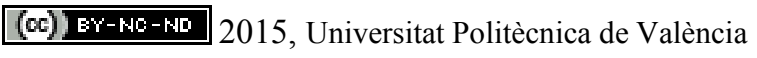


- La mayoría (un 86\%) de los alumnos consideraba que los puentes pueden ser considerados obras de arte.

- Un $47 \%$ de los alumnos estaba totalmente de acuerdo con la afirmación "Los puentes bonitos son caros". El resto (un 53\%) estaba algo de acuerdo con la afirmación anterior.

- Todos los alumnos estaban totalmente de acuerdo o algo de acuerdo en considerar la pasarela de la Arganzuela (Fig. 4) como un buen diseño. Este es un ejemplo de una estructura en la que la estética ha jugado un papel primordial, pero que ha sido poco económica en comparación con proyectos similares. Por tanto, es un ejemplo que apoya la idea general de que "los puentes bonitos son caros". No obstante, los alumnos no tenían ningún dato referente al coste de la estructura cuando se les pidió que opinasen sobre su calidad.

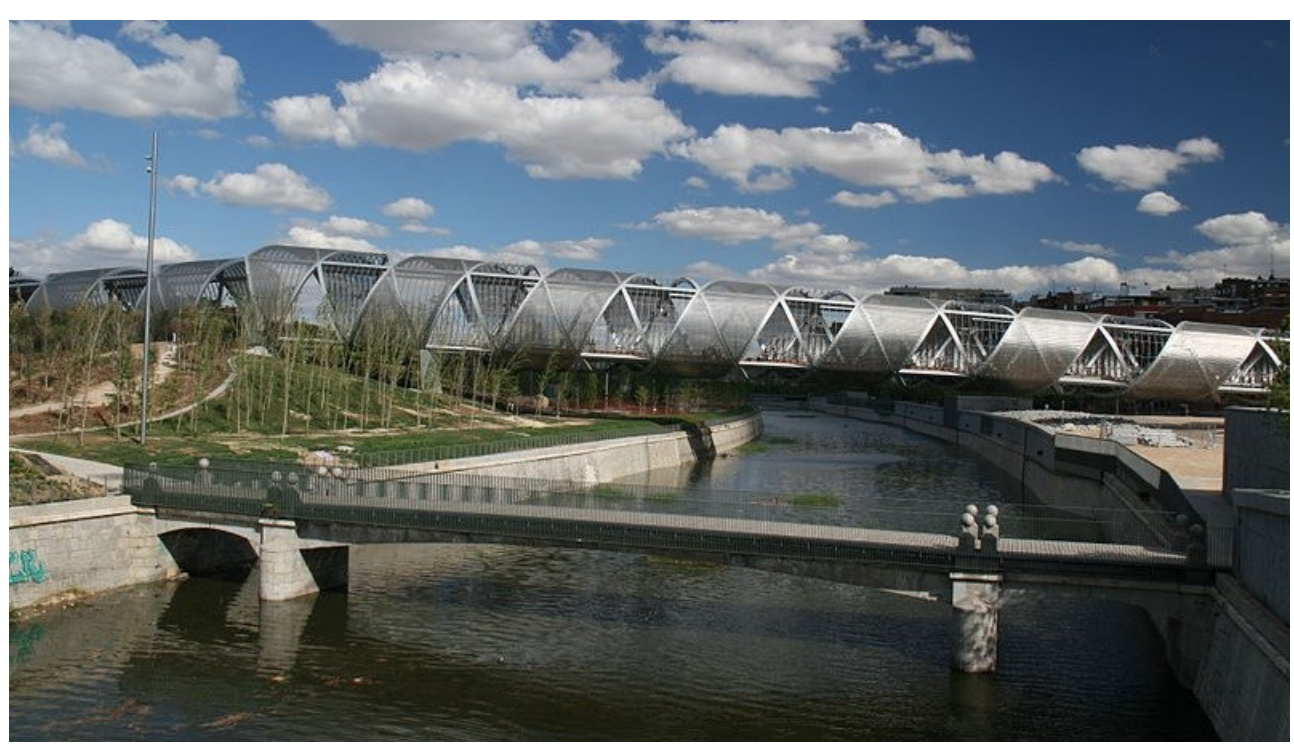

Fig. 4. Pasarela de la Arganzuela (en el fondo) en Madrid. Arq. D. Perault, Ing. MC2. Imagen: Tamorlan / Wikimedia Commons / CC-BY-SA-3.0.

El análisis de estos resultados revela que la opinión general es que los puentes los diseñan principalmente los ingenieros y que pueden ser obras de arte. Sin embargo, los alumnos piensan que la estética es fundamentalmente obra de los arquitectos y que la belleza resulta cara. Estas opiniones son bastante comunes entre la sociedad, pero no por ello son necesariamente ciertas. "Desmontar" estos mitos es algo que se intentó realizar a lo largo del resto de la actividad.

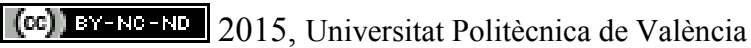


"¡Abre los ojos, un puente!" o cómo tomar conciencia de la importancia de la ingeniería mientras se aprende un idioma extranjero

b) Fase 2. Selección por los alumnos de un puente que les gustaba mucho y explicación razonada del por qué de su elección. El objetivo de esta fase era propiciar la reflexión del alumno y el que se expresara públicamente en español.

c) Fase 3. Construcción por parte de los alumnos de modelos reducidos de puentes con un folio de papel (Fig. 5). Todos los puentes debían salvar la misma distancia (alrededor de $10 \mathrm{~cm}$ ) y resistir una carga determinada por los profesores (diez chocolatinas). A través de esta actividad, los alumnos podían tomar consciencia de lo importante que es la forma, la distancia entre apoyos y el canto en la resistencia de una estructura.

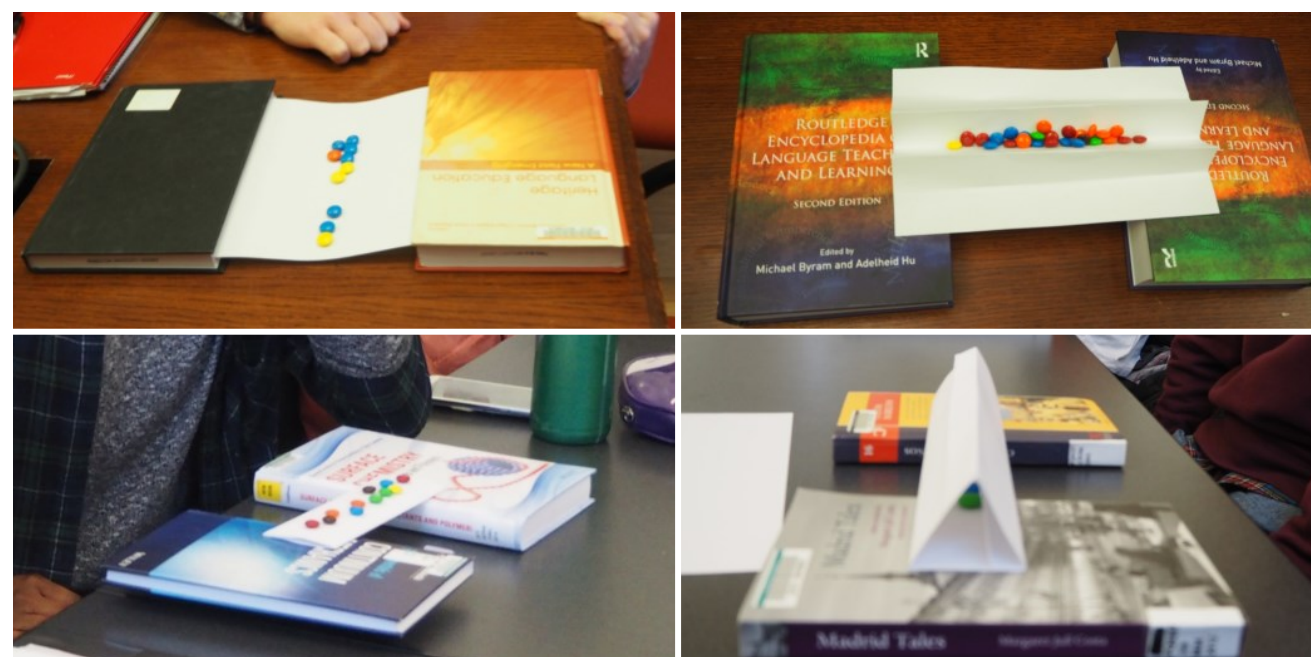

Fig. 5. Ejemplos de modelos reducidos de puentes construidos por los alumnos.

d) Fase 4. Realización de una presentación interactiva y dinámica en la que se explicaban:

- Los principales conceptos de la ingeniería estructural.

- Las principales ideas que permiten valorar los puentes como obras de arte.

- La dimensión social de los puentes o por qué es importante que los ciudadanos comprendan su valor.

Puesto que los alumnos deben analizar elementos de las ciudades de Buenos Aires y de Madrid, la presentación incluía muchos puentes de esas ciudades. Algunos de los ejemplos empleados fueron:

- El Puente de la Mujer (Fig. 6) y el Puente Alsina en Buenos Aires. 
- Los puentes sobre el anillo ciclista y las pasarelas cáscara en Madrid (Fig. 7).

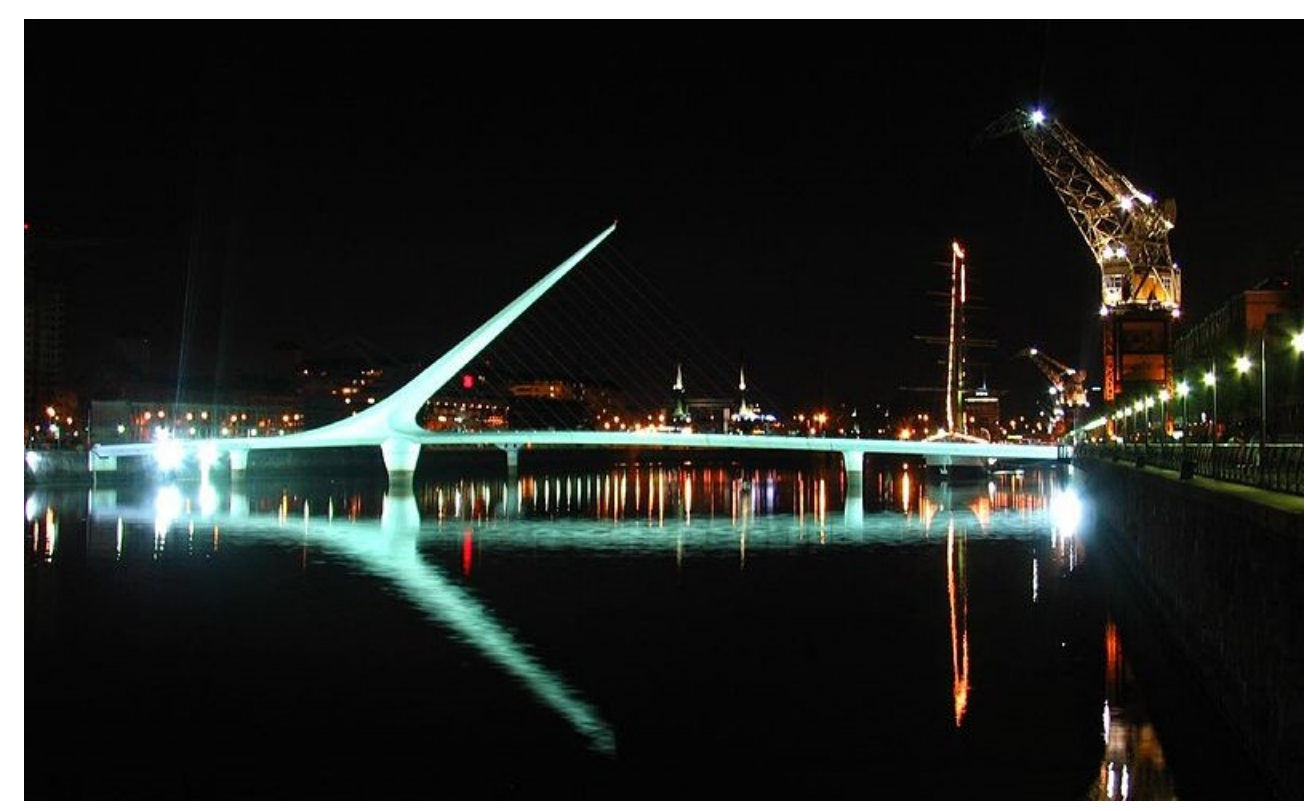

Fig. 6. Puente de la Mujer en Puerto Madero, Buenos Aires (2001). Proyectista: S. Calatrava. Imagen: A. González Molina / Wikimedia Commons / CC-BY-SA-3.0.

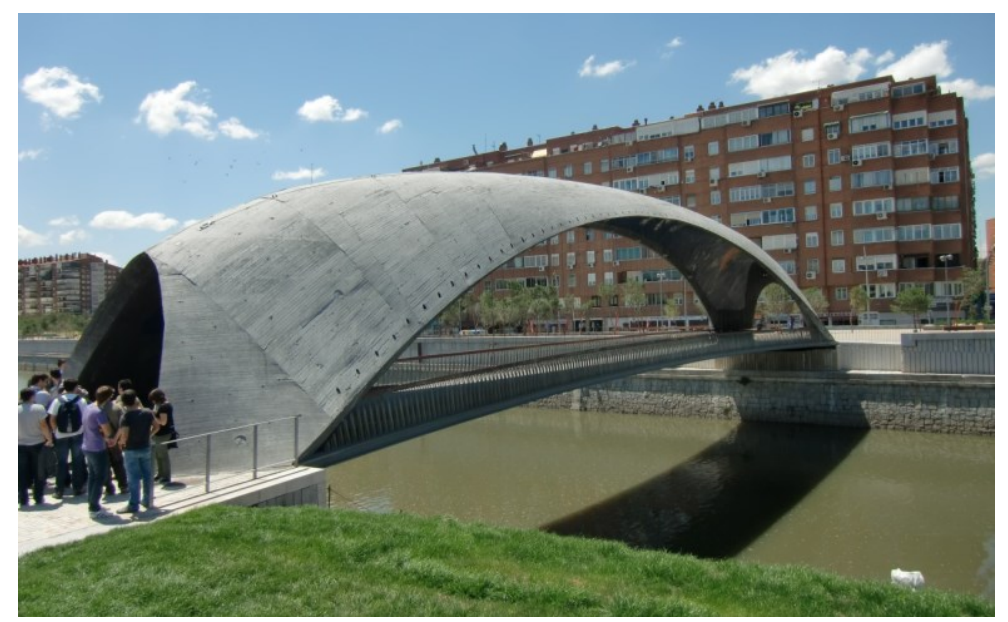

Fig. 7. Pasarela Cáscara en el proyecto Madrid Río, Madrid (2010). Arquitectura: Equipo Madrid Río, ingeniería: Fhecor Ingenieros Consultores. Imagen: I. Payá Zaforteza.

Además, la presentación intercaló algunos fragmentos de un video que contenía una entrevista con Javier Manterola (véase http://spanishbridges.princeton.edu/films.html en

\section{(cc) BY-NG-ND 2015, Universitat Politècnica de València}

Congreso In-Red (2015) 
"¡Abre los ojos, un puente!” o cómo tomar conciencia de la importancia de la ingeniería mientras se aprende un idioma extranjero

Payá-Zaforteza y Garlock, 2015), un destacado ingeniero español y antiguo catedrático de puentes de la Escuela de Ingenieros de Caminos de Madrid. Javier Manterola es autor de, entre otros, el proyecto del nuevo puente sobre la Bahía de Cádiz (Fig. 8), también conocido como el puente de La Pepa, que es el puente de mayor luz (distancia entre apoyos) de España. En estos fragmentos de entrevista, el profesor Manterola explicaba cómo influía la estética en sus diseños y daba consejos (a petición de los entrevistadores) sobre cómo llegar a ser un gran proyectista de puentes.

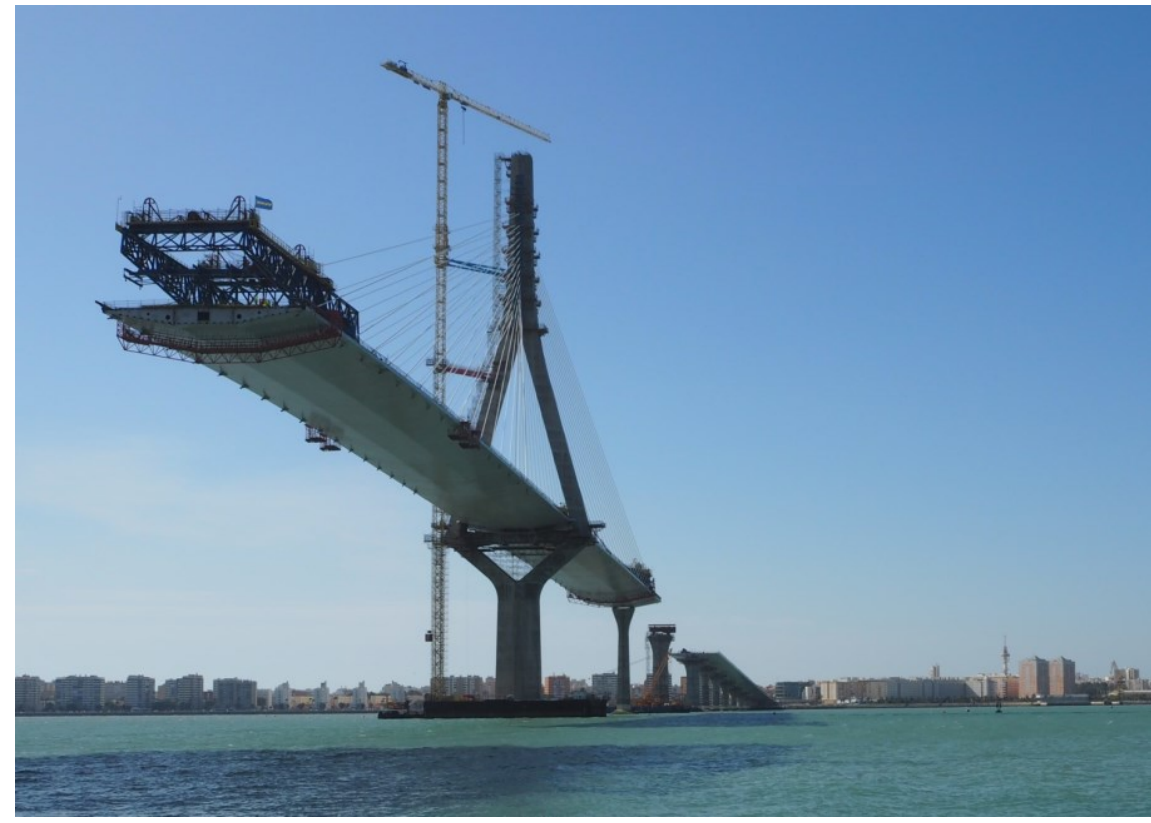

Fig. 8. Fotografía del nuevo puente sobre la Bahía de Cádiz tomada durante su construcción. Imagen: I. Payá Zaforteza.

\section{Resultados}

La actividad ha tenido una acogida muy positiva entre el alumnado. Esta afirmación se apoya en la actitud de los alumnos durante la actividad, en los comentarios personales realizados a los profesores firmantes del artículo y, muy especialmente, por la inclusión de algunos ejemplos de puentes en los portfolios digitales de los alumnos. Por ejemplo, un grupo de estudiantes incluyó en su portfolio el puente transbordador del Riachuelo Nicolás Avellaneda, en la ciudad de Buenos Aires. Este portfolio demuestra el impacto de la actividad, ya que sus autores retoman ideas y conceptos presentados durante la charla, apropiándose de ellos para hacer un análisis crítico de la manera en que este puente en particular responde a los criterios puestos a su consideración (elegancia, eficiencia, economía -también conocidos como "las tres e's"). Desde el punto de vista del aprendizaje, 
el portfolio también demuestra el cumplimiento de los objetivos de ampliar y reutilizar el vocabulario en nuevos contextos (los alumnos se refieren al puente como "un monstruo de hierro" con un "estilo industrial y robusto", etc.) y de desarrollar una nueva mirada hacia los puentes, que pasan a ser analizados críticamente ("uno de los tres e's, la elegancia no es muy evidente (...) debido a su estilo industrial y robusto"). Es necesario indicar que los profesores tenían previsto pasar al final de la actividad de nuevo el cuestionario realizado con la aplicación "Kahoot" y comparar los resultados obtenidos para medir objetivamente el impacto conseguido, pero las limitaciones del tiempo disponible no lo hicieron posible.

A través de la actividad los alumnos han tenido además la oportunidad de mejorar su comprensión oral del español al entablar un contacto directo con un profesor hispanoparlante, han podido incorporar nuevos términos a su vocabulario de español y han tomado conciencia de que ese idioma, y no solo el inglés, es empleado para producir y transmitir conocimiento científico. Estas competencias se han adquirid en una situación similar a la de un congreso, conferencia o reunión, que son eventos en los que los participantes escuchan a un ponente y toman notas, debaten, y responden a preguntas de forma justificada.

La actividad ha sido muy positiva también para los profesores participantes, pues ha permitido un enriquecimiento mutuo y supone un punto de partida para experiencias docentes más complejas que integren el aprendizaje de un idioma extranjero con la ingeniería y el arte.

\section{Conclusiones}

La ingeniería es vista en muchas ocasiones como una disciplina estrictamente técnica, carente de la creatividad artística de la música o la arquitectura. Además, la ingeniería estructural muchas veces es vista como algo muy complejo y por ello poco atractivo para los no doctos en la materia. Sin embargo, sus principios básicos son muy intuitivos, se pueden aprender fácilmente y permiten abrir los ojos hacia una nueva forma de ver la ingeniería que incorpora el arte y la creatividad como esta actividad ha demostrado.

Por su parte, el aprendizaje de lenguas tiende a evitar la complejidad en los temas y discursos abordados partiendo de la noción que los alumnos no poseen las herramientas lingüísticas necesarias para procesar tal complejidad. Con esta experiencia contribuimos a mostrar que, al contrario, los estudiantes universitarios están preparados para adquirir algo más que el lenguaje de las interacciones cotidianas en contextos informales. Ser capaces de percibir la lengua en contextos profesionales, en relación con disciplinas como la ingeniería civil, los acerca a escenarios y situaciones en las que se encontrarán quienes prolonguen su contacto con el español en el marco de sus estudios (por ejemplo, a través de viajes de estudio y/o prácticas en un país hispanohablante) o más adelante, en su vida profesional.

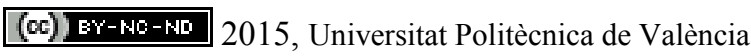

Congreso In-Red (2015) 
"¡Abre los ojos, un puente!” o cómo tomar conciencia de la importancia de la ingeniería mientras se aprende un idioma extranjero

En resumen, esta ponencia ha presentado una actividad en la que los alumnos participantes desarrollan una nueva mirada hacia la ingeniería estructural mientras desarrollan competencias lingüísticas relativas al dominio de un idioma extranjero. Además, es un ejemplo de colaboración multidisciplinar que puede proporcionar ideas e inspiración para desarrollar proyectos similares en un futuro.

\section{Referencias}

BILLINGTON, D.P. (1985). The Tower and The Bridge. The New Art of Structural Engineering. Princeton, NJ, USA: Princeton University Press.

BILlington, D.P. (2013). La Torre y el Puente. El Nuevo Arte de la Ingeniería Estructural. Madrid: Cinter Divulgación Técnica.

BYRNES, H. (2002). The Cultural Turn in Foreign Language Departments: Challenge and Opportunity. Profession, 114-129.

MODERN LANGUAGE ASSOCIATION (2007). Foreign Languages and Higher Education: New Structures for a Changed World. Profession 234-245. < https://www.mla.org/flreport> [Consulta: 28 de mayo de 2015].

NATIONAL STANDARDS IN FOREIGN LANGUAGE EDUCATION PROJECT (2006). Standards for Foreign Language Learning in the 21st Century (3rd Ed.). Lawrence, KS, USA: Allen Press.

PAYÁ-ZAFORTEZA I., GARLOCK M.E.M. The Art of Spanish Bridge Design. $<$ http://spanishbridges.princeton.edu $>$ [Consulta: 28 de mayo de 2015].

SOBRINO J. (2013). "A Bridge is More Than a Bridge: Aesthetics, Cost and Ethics in Bridge Design”. Structural Engineering International 23(3): 340-345. 